\title{
Optimizing Support Vector Machine Parameters based on Quantum and Immune Algorithm
}

\author{
Yuling Tian* \\ College of Computer Science and Technology, Taiyuan University of Technology, Taiyuan, 030600, China
}

\begin{abstract}
In view of premature convergence and blind searching of the quantum and immune algorithm in the evolution process, this paper proposes two improvements. Firstly, the fitness function is improved by utilizing the mean square error as the fitness function, and the concentration of immune antibodies is introduced to the fitness function to improve the diversity of populations and avoid premature convergence of the algorithm. Secondly, the probability of rotation is adopted to optimize the quantum rotate gate to avoid blind searching and accelerate the convergence of the algorithm. The improved algorithm is adopted to optimize parameters of support vector machines and is applied to network intrusion detection. The experimental results show that the improved algorithm has better optimization effects.
\end{abstract}

Keywords: support vector machine; parameter optimization; quantum computation; quantum immune algorithm

(Submitted on October 23, 2018; Revised on November 26, 2018; Accepted on December 28, 2018)

(C) 2019 Totem Publisher, Inc. All rights reserved.

\section{Introduction}

Support vector machines (SVM) are widely used in the field of machine learning and was proposed by Vapnik et al. in 1995 [1]. It is based on the statistical learning theory and structural risk minimization principle and has advantages in solving the small sample problem, nonlinearity, and high dimension. It also shows great generalization ability. However, a typical problem of SVM is the selection of model parameters, which is closely related to the performance of SVM. Specific sample data shows that the performance of SVM is mainly affected by kernel function parameters and penalty factors. Different parameter settings make the accuracy of the model very different. Improper parameter settings may lead to under-fitting and over-fitting problems of the model. Therefore, it is of great significance and value to study SVM and optimize the parameters of SVM in practical applications.

Parameter optimization is widely studied in many applications associated with SVM. For example, Liu proposed an adaptive niche quantum-inspired immune clonal algorithm for making the multi-modal function more effectively and converge to extreme value points based on the quantum coding, immune clone, and niche mechanism [2]. Demidova presented an improved particle swarm optimization algorithm for searching the kernel function type, kernel function parameter, and regularization parameter simultaneously [3]. Ikram optimized the selection of kernel parameters utilizing the automatic parameter based on principal component analysis and SVM [4]. Wang et al. introduced the multiclass support vector machine (MSVM) with parameters optimized by particle swarm optimization to achieve an anomaly-based intrusion detection [5]. Rong adopted the genetic algorithm to optimize the parameters of SVM for higher classification accuracy [6]. Liu et al. optimized the parameters of SVM based on a strategy of stepwise optimizing parameters of the contour plots of cross-validation accuracy and shortened the time of parameter optimization remarkably [7]. In paper [8], PCA was used to extract principal components from the evaluation indexes of various oxides, and then the genetic algorithm was used to optimize the parameters of SVM for higher classification. Mei presented a modified classifier using the improved particle swarm optimization to optimize the parameter of SVM based on the new dynamic inertia weight, global neighborhood search, shrinkage factor, and mutation operator of the genetic algorithm [9].

\footnotetext{
* Corresponding author.

E-mail address: tianyuling@tyut.edu.cn
} 
This paper presents a parameter optimization algorithm of SVM based on the quantum and immune algorithm. The quantum and immune algorithm is used to solve the problem of parameter optimization of SVM. However, the traditional algorithm is no longer able to solve the premature convergence and blind searching problem in the evolution process. It is necessary (and urgent, considering the current demand) to improve the optimization method based on the quantum and immune algorithm that can work sufficiently quickly. Therefore, to solve these problems, this paper proposes two improvements to achieve better optimization effects and effectiveness.

\section{A Synopsis of Support Vector Machine Parameters}

\subsection{Basic Mathematical Forms of Support Vector Machine}

Given the training dataset [10] $T=\left\{\left(x_{1}, y_{1}\right), \cdots,\left(x_{n}, y_{n}\right)\right\}$ :

$$
\begin{gathered}
\min _{w, b, \xi}\|\omega\|^{2} / 2+C \sum_{i=1}^{n} \xi_{i}^{2} \\
\text { s.t. } \quad y_{i}\left(\omega^{T} x_{i}+b\right) \geq 1-\xi_{i}, \text { for } i=1, \cdots, n \\
\xi_{i} \geq 0, \text { for } i=1, \cdots, n
\end{gathered}
$$

Where $x_{i} \in R^{n}, y^{i} \in\{1,-1\},(i=1, \cdots, n) ; C$ is the penalty parameter, which is greater than 0 ; $\omega$ is the weight vector; $\xi$ is the slack variable; and $b$ is the threshold of weight. Dealing with general classification problems, the input space is mapped to a high-dimensional Hilbert space called $H$, the transform of $x=\phi(x)$, and then the best hyperplane for classification and the corresponding decision function, $f(x)=\operatorname{sgn}(\langle w, \phi(x)\rangle+b)$, are found in $H$, the feature space. $w$ and $b$ can be obtained using Equation (1).

\subsection{The Support Vector Machine Parameters}

The selection of various parameters in support vector machines, such as the penalty parameter $C$, the loss function, the kernel function, and its parameters $g$, have very important effects on the learning ability and generalization ability of the learning machine. At present, the usual methods are the grid method, simulated annealing algorithm, genetic algorithm, immune algorithm, and other intelligent algorithms. Most of these methods are applied in the classification of SVM.

The penalty parameter, $C$, is one of the important parameters of SVM. It represents the degree of punishment to a training model, which makes the allocation of error samples and is used to compromise the approximation error and the complexity of the model, that is, to control the balance between the maximization of the classification interval and the minimization of the training error. The larger the penalty parameter $C$, the higher the fitting degree of the data and the more stable the empirical risk and generalization ability of the training model, but the worse the generalization ability of the SVM model. This transforms into the traditional empirical risk minimization. When the penalty parameter $C$ becomes lower, the punishment of empirical error will be lower and the training error will be relatively higher; in this condition, the learning machine will be relatively simpler, but the empirical risk will become higher. The penalty parameter monitors the weight of empirical risk and confidence intervals of the learning machine in order to possess a better learning ability. The value of $C$ cannot be too big or too small, because the appropriate penalty parameter $C$ can resist interference to a certain extent and affects the processing of outliers in samples. Therefore, to ensure the accuracy and stability of the SVM model, the penalty parameter should be reasonably selected.

The kernel function with its adjustment function is one of the most important functions of the SVM model and can determine the nonlinear mapping between the high dimensional space and input space and between the type and complexity of classifiers. If different kernel functions are selected, that is, different mapping functions are selected, the complexity of the training sample subspace distribution is different. In the subspace, the maximum dimension of the linear classification surface is determined by the dimension of the sample data subspace, that is, the minimum empirical error is determined by the sample data subspace. The sample data subspace and the best classification hyperplane are corresponding; therefore, if the dimension of the subspace is higher, the generalization ability of the classification hyperplane may be more complex, the confidence 
interval will be larger, and the empirical risk will be lower. At present, there are few theories on the selection of kernel function. The usual method is to select parameters and the type of classifiers based on prior knowledge or to optimize them step by step in the training. In short, selecting the appropriate kernel function is essential, and then the training samples will be mapped to the appropriate feature space so the SVM model with better generalization performance will be obtained.

The parameter $g$ of the kernel function mainly affects the complexity of the distribution of sample data in highdimensional feature space. For a certain $\mathrm{C}$ that is large enough, it is easy to overlearn when $g$ is close to zero. The SVM classifier could correctly separate training samples, but it does not have any generalization ability to test samples. When $g$ approaches infinity, all training samples are classified by the SVM classifier to the class with larger number of samples. The generalization ability of the SVM classifier becomes almost zero, that is, the phenomenon of underlearning.

The training error and generalization ability of the SVM model are determined by the parameters of the kernel function $g$ and the penalty parameter $C$, as well as the complexity of data sample feature space and the performance of SVM. In practical applications, the selection of the kernel function parameter $g$ in SVM is equally important or even more important than the kernel function selection. For the SVM based on a certain kernel function, different SVM models can be obtained by selecting a different kernel function parameter $g$ and penalty parameter $C$, and the two parameters influence the diagnosis of the model to a large extent. Therefore, in order to obtain the relatively optimal diagnostic effects, selecting appropriate parameters is essential.

\section{The Support Vector Machine Parameters Optimization Method based on Quantum and Immune Algorithm}

The SVM parameters $(C, g)$ are defined as the quantum antibody, and the population of quantum antibodies is represented by $Q(t)=\left\{q_{1}^{t}, q_{2}^{t}, \cdots, q_{n}^{t}\right\}$, where $t$ represents iterations, $n$ represents the population size, and the quantum antibody, $q_{j}^{t}$, is represented by the following:

$$
q=\left[\begin{array}{cccccc}
\alpha_{1} & \alpha_{2} & \cdots & \beta_{i} & \cdots & \alpha_{m} \\
\beta_{1} & \beta_{2} & \cdots & \alpha_{i} & \cdots & \beta_{m}
\end{array}\right]
$$

Where $m$ represents the length of the quantum antibody, which is the number of qubits, and $j=1,2, \cdots, n$.

Firstly, initialize the quantum antibody population, produce $N$ antibodies at random, where each of them is in a linear superposition state of all possible states with the same probability, $1 / \sqrt{2^{m}}$, and the population size is $N$. Initialize both $\alpha_{i}^{t}$ and $\beta_{i}^{t}$ of the quantum antibody $q_{j}^{t}$ as $1 / \sqrt{2}$, that is, in the initial state, every antibody is in a linear superposition state of all possible states with the same probability, $1 / \sqrt{2^{m}}$, as shown in Equation (3):

$$
\left|\varphi_{q j}^{0}\right\rangle=\frac{1}{\sqrt{2^{m}}} \sum_{k=1}^{2^{m}}\left|s_{k}\right\rangle
$$

Where $s_{k}$ represents the $k^{\text {th }}$ state of antibody, $x_{i}=0,1,(i=1,2, \cdots, m)$.

Secondly, observe all individuals, generate the binary solution set $P(t)=\left(p_{1}^{t}, p_{2}^{t}, \cdots, p_{m}^{t}\right)$. Each solution is a $m$ dimensional vector and a binary string of $m$-length, and its value is determined by the corresponding observation probability, $\left|\alpha_{i}^{t}\right|^{2}$ or $\left|\beta_{i}^{t}\right|^{2}$, where $i=1,2, \cdots, m$. That is, firstly, generate a number between 0 and 1 at random. If the number is greater than $\left|\alpha_{i}^{t}\right|^{2}$, the bit value is 1 , and if it is less, then the bit value is 0 .

Thirdly, calculate the fitness of each solution in $P(t)$, record the individual with the best fitness, and the optimal solution is stored as the target value of the next generation of evolution. This paper utilizes the mean-square error (MSE) as the fitness function, and the formula is shown as Equation (4):

$$
M S E=\frac{1}{N} \sum_{i=1}^{N}\left(y_{i}-\hat{y}_{i}\right)^{2}
$$


Where $N$ is the number of samples, $y_{i}$ is the actual value, and $\hat{y}_{i}$ is the predictive value.

Fourthly, clone and select quantum antibodies. Utilize the quantum rotate gate to clone and amplify the quantum antibody for changing the angle of the qubit of the antibody. To realize the clonal expansion of excellent antibodies, the quantum rotate gate angle changes in the local optimization traversal of the small region. The general range of it is $(0.005 \pi, 0.1 \pi)$, and to make it similar to the maternal antibody so as to achieve the effect of cloning and amplification, we set it as $0.01 \pi$ in this paper. The rotation angle is arbitrary and helps to improve the antibody diversity. The number of the clonal expansion is determined by Equation (5). Firstly, calculate the fitness of the antibody population, and then sort the fitness function of this paper according to the fitness value. The best fitness function is the fitness function with the minimum value.

$$
m_{i}=\operatorname{round}\left(\frac{\beta \times N}{i}\right), i=1,2, \cdots, N
$$

Where $m_{i}$ represents the clone size of the $i^{\text {th }}$ antibody in fitness order, $\beta$ is the multiplier factor that determines the scale, and $N$ is the size of the population.

Fifthly, use the quantum not-gate to mutate the quantum antibody. The essence of the quantum not-gate is to implement the operation of the quantum rotate gate on qubit. The angle of rotation direction is certain with a fixed size, $\pi / 2$, and it makes a reversal of the probability amplitude over a qubit. Thus, it can also be used in other large angles to implement the mutation operation on qubits. Select the antibody with poor fitness, choose qubits at random, if determined, and then operate the implementation of quantum not-gate to exchange the probability amplitude of some qubits of the antibody and realize the mutation operation of the quantum antibody in Equation (6):

$$
\begin{array}{r}
X=\left[\begin{array}{ll}
0 & 1 \\
1 & 0
\end{array}\right] \text { represents the quantum not-gate, to mutate the } i^{\text {th }} \text { qubit, }\left[\begin{array}{c}
\alpha_{i} \\
\beta_{i}
\end{array}\right] \\
X\left[\begin{array}{c}
\alpha_{i} \\
\beta_{i}
\end{array}\right]=\left[\begin{array}{ll}
0 & 1 \\
1 & 0
\end{array}\right]\left[\begin{array}{c}
\alpha_{i} \\
\beta_{i}
\end{array}\right]=\left[\begin{array}{c}
\beta_{i} \\
\alpha_{i}
\end{array}\right]
\end{array}
$$

After the mutation operation, the antibody is defined as Equation (2).

Sixthly, calculate and update the fitness of the population. Compare the optimal solution in current with the stored optimal solution. If the stored optimal solution is inferior to the current optimal solution, then it will be replaced by the current optimal solution and the current optimal solution will become the target value of the next iteration at the same time. Otherwise, keep the stored optimal solution unchanged. The algorithm ends until the condition is satisfied or the maximum iterations is reached. In the iterative process of the algorithm, the solution of the population evolves to the optimal solution gradually and finally converges. The flow chart of the algorithm is shown in Figure 1.

\section{Improvement of the Algorithm}

The classification accuracy of the SVM optimized by the quantum and immune algorithm (QIA) is higher than that of the SVM optimized by the grid search method or the immune algorithm. The learning ability and generalization ability of the QIA algorithm are better than those of the other algorithms. When the number of training samples sets is different, the simulation results of SVM optimized by the quantum immune algorithm cannot possess the best consistency, which is the result of premature convergence in the evolution of the algorithm and the random selection of qubits and causes some individuals to regress to a certain extent. Therefore, this section further improves the quantum immune algorithm to improve the learning ability and generalization ability of SVM.

\subsection{Improved Quantum and Immune Algorithm}

Optimization of the kernel function parameters and penalty parameters of SVM by adopting the quantum immune algorithm [11] could improve the performance of support vector machines. The optimization also has some shortcomings, that is, premature convergence and blind problems in the process of evolution. In this section, some improvements are made to the 
quantum immune algorithm, and an improved SVM parameters optimization method based on the quantum and immune algorithm is presented.

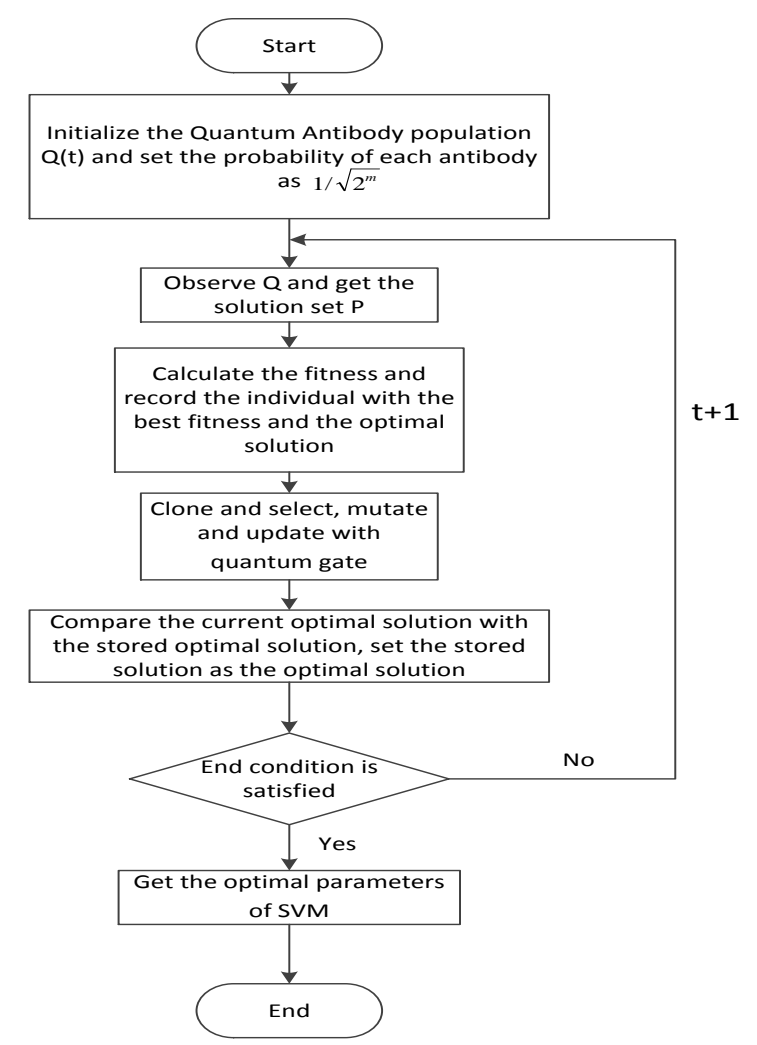

Figure 1. The flow chart of the support vector machine parameters optimization algorithm

\subsubsection{Fitness Function}

This paper selects the mean square error as the fitness function, which usually measures the error between the predicted value and the actual value. The smaller the mean square error, the closer the predicted value is to the actual value. To improve the diversity of the population and suppress the premature convergence in the evolution process, this paper introduces the concentration of antibodies as a part of the fitness function. The fitness should be as small as possible.

Before defining the fitness function, the following mathematical descriptions are given [12]:

(1) Information entropy of antibody

In the immune algorithm based on information entropy, the population $G$ is a set of $N$ strings of $M$-length. Supposing there are $N$ antibodies, the information entropy of its loci $m, H_{m}(N)$, is expressed as Equation (7):

$$
H_{m}(G, N)=\sum_{k=1}^{M}-P_{k m} \log _{2} P_{k m}
$$

Where $N$ is the number of antibodies, $P_{k m}$ is the probability on the loci $\mathrm{m}$ where the $k^{\text {th }}$ symbol appears, that is, $P_{k m}=$ the total number of $k^{\text {th }}$ symbols appearing on locus $m / N$.

\section{(2) Similarity}

Similarity refers to the degree of similarity between two antibodies $i$ and $j$ or between antibody $i$ and antigen $j$, recorded as $A_{i, j}$, as shown in Equation (8):

$$
A_{i, j}=1 /(1+H(G, 2)), G=(i, j)
$$


The larger the $A_{i, j}$, the higher the similarity between $i$ and $j$. Here, $A_{i, j}=1$ means that $i$ and $j$ have exactly the same genetic value, and the range of $A$ is between 0 and 1 .

(3) Antibody concentration

Antibody concentration refers to the proportion of antibodies and their similar antibodies in the population, and the concentration of antibodies can be defined as Equation (9):

$$
d_{i}=P\left(A_{i, t}\right) / N
$$

Where $A_{i, t}$ represents the number of antibodies whose similarity with antibody $t$ is greater than $\lambda$. $\lambda$ usually has a value of $0.9 \leq \lambda \leq 1$ and represents the similarity constant.

The antibody fitness and antibody concentration are evaluated comprehensively, the fitness of the antibody is adjusted, and the improved fitness, which is the result of the interaction of antibody concentration and fitness, is obtained. It can be defined as Equation (10):

$$
f_{i}^{\prime}=f_{i} \times \exp \left(k \times d_{t}\right)
$$

Where $d$ is the antibody concentration. It can be seen from the above formula that the new fitness $f_{i}^{\prime}$ is obtained by modifying the antibody fitness $f_{i}$. In solving the minimization problems, the parameter $k$ represents a positive constant adjustment factor, whose value is determined by the size and experience of the evolutionary population. The improved fitness is selected as the fitness of the antibody. That is, when the individual proliferates, the probability of being selected is higher if the fitness or the concentration of antibody is low. In this way, the diversity of individuals is maintained, the antibody of excellent original fitness is retained, and the selection pressure of similar antibodies is reduced [12].

In the evolution of the quantum and immune algorithm, the improved fitness is selected as the fitness function of this algorithm. Through this way, it can not only avoid the premature convergence of the algorithm, but also maintain the diversity of the antibodies.

\subsubsection{Improvement of Quantum Rotate Gate}

In the quantum evolutionary algorithm, the quantum rotate gate is used to update the quantum states, and its transformation matrix and transformation formula are shown as Equations (11) and (12):

$$
\begin{gathered}
U(\theta)=\left[\begin{array}{cc}
\cos \theta & -\sin \theta \\
\sin \theta & \cos \theta
\end{array}\right] \\
{\left[\begin{array}{c}
\alpha_{i}^{\prime} \\
\beta_{i}^{\prime}
\end{array}\right]=U\left(\theta_{i}\right)\left[\begin{array}{c}
\alpha_{i} \\
\beta_{i}
\end{array}\right]=\left[\begin{array}{cc}
\cos \left(\theta_{i}\right) & -\sin \left(\theta_{i}\right) \\
\sin \left(\theta_{i}\right) & \cos \left(\theta_{i}\right)
\end{array}\right]}
\end{gathered}
$$

Where Equation (11) represents the quantum bit of the updated $i^{\text {th }}$ bit, Equation (12) represents the quantum bit of the $i^{\text {th }}$ bit before updating, $\theta$ is the angle of rotation, $\theta_{i}=s\left(\alpha_{i}, \beta_{i}\right) \Delta \theta_{i}, \Delta \theta_{i}$ is the degree of the angle of rotation to adjust the convergence rate of the algorithm, and $s\left(\alpha_{i}, \beta_{i}\right)$ is the direction of rotation, which is used to guarantee the convergence direction of the algorithm. The angle and direction of the rotation determine the performance of the algorithm.

Here, we need to ensure the diversity of populations in the pre-evolutionary period, and to prevent individuals from regressing, we introduce the concept of rotation probability. The formula of rotation probability $P$ is shown as Equation (13):

$$
P=e^{t / T-1}
$$


Where $T$ is the total number of iterations and $t$ is the current number of iterations. It can be seen from the formula that the rotation probability $P$ is an increasing function, and its value ranges is from 0 to 1 .

In the evolution process of the unimproved quantum and immune algorithm, the individual rotates towards the global optimal value in each iteration process, which is unfavorable to the individual difference in the early stage of the algorithm. Therefore, if the probability rotation is introduced, the individual could approach the global optimal value probabilistically and rotate towards the average value of the population. In this way, a coarse-grained searching is carried out at the initial stage of the algorithm, so that more quantales are closer to the average location of the population. Then, as the number of iterations increases, the probability that the individual rotates towards the global optimal value increases. Therefore, the introduction of probability rotation can speed up the convergence of the algorithm and avoid retrogression of some individuals.

\subsection{Experimental Validation of Test Functions}

We verified the performance of the improved quantum immune algorithm by using three typical test functions and compared it with the unimproved quantum immune algorithm. The minimum values of the three test functions were calculated respectively, and the minimum value was 0 . Because the dimensions of the test function have little impact on the test results, we selected $n=3$ here. The test functions are as follows:

The Rastrigin function is shown in Equation (14):

$$
f(x)=\sum_{i=1}^{n}\left(x_{i}^{2}-10 \cos \left(2 \pi x_{i}\right)+10\right),-5.12 \leq x_{i} \leq 5.12
$$

The Griewank function is shown in Equation (15):

$$
f(x)=\sum_{i=1}^{n} \frac{x_{i}^{2}}{4000}-\prod_{i=1}^{n} \cos \left(\frac{x_{i}}{\sqrt{i}}\right)+1, \quad-600 \leq x_{i} \leq 600
$$

The Rosenbrock function is shown in Equation (16):

$$
f(x)=\sum_{i=1}^{n-1}\left(100\left(x_{i+1}-x_{i}^{2}\right)^{2}+\left(x_{i}-1\right)^{2}\right),-2.048 \leq x_{i} \leq 2.048
$$

The test results of the test function are shown in Figures 2, 3, and 4.

The test results of the three test functions show that the improved quantum immune algorithm has better convergence performance than the unimproved quantum immune algorithm, which indicates that the improved quantum immune algorithm improves the performance of the quantum immune algorithm as a whole.

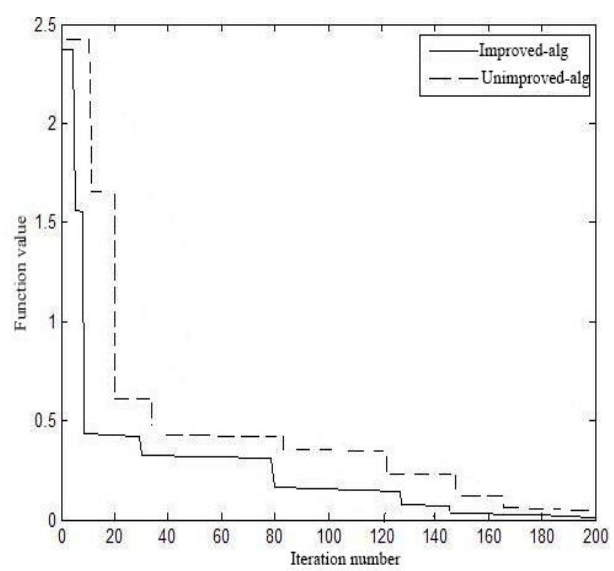

Figure 2. The test results of Rastrigin function 


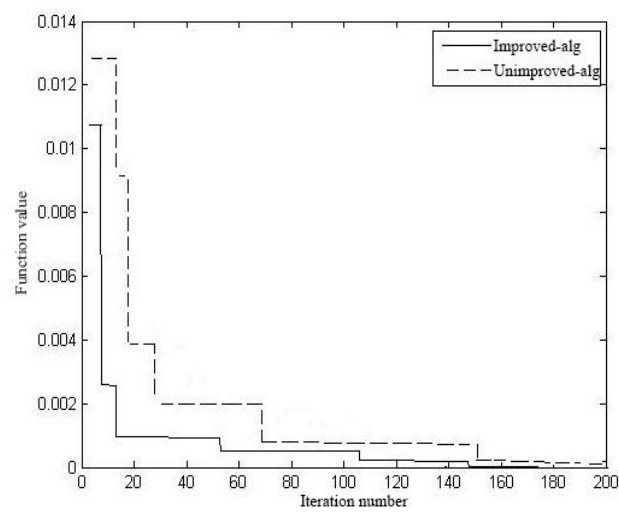

Figure 3. The test results of Griewank function

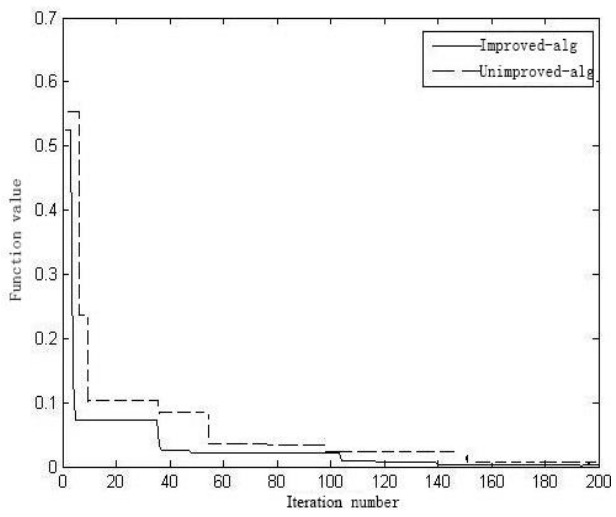

Figure 4. The test results of Rosenbrock function

\subsection{An Improved Support Vector Machine Parameters Optimization Algorithm based on Quantum and Immune}

In this section, the fitness function and quantum rotate gate of the quantum and immune algorithm are improved, and the steps of parameters optimization of SVM by the improved algorithm are as follows:

(1) Construct an initialized population, $Q(t)$, and set the iterations $t=0$.

(2) After the quantum-state collapse, $Q(t)$ produces the observed population $P(t)$. Use the improved fitness function to calculate the fitness of each individual in the current population. Record the optimal value of the current population, and store the optimal solution as the target value of the next generation evolution.

(3) Use the improved quantum rotate gate to clone and select, and then carry out the mutation operation to obtain the new population $Q^{\prime}(t)$.

(4) Calculate the fitness of the updated population, and compare the current optimal solution and the stored optimal solution to obtain the optimal solution to store.

(5) Determine whether to meet the stop condition. If it is, then stop the algorithm and output the result of the operation; otherwise, turn to step (2).

\section{Application of the Algorithm}

\subsection{Experimental Data}

We used the authoritative KDD CUP 1999 intrusion detection dataset to conduct our verification experiment. The KDD CUP 1999 intrusion detection dataset was established by an intrusion detection evaluation project of the DARPA (Defense Advanced Research Projects Agency) and formed by Wenke Lee, who extracted as much security information as possible from a large number of redundant information data. This dataset is a simulation of US Air Force LAN, including the simulation of various service hosts and various types of users. It not only includes normal network flow, but also includes several intrusive network attacks in the Air Force network. In 1999, the dataset was used in the KDD (Knowledge Discovery and Data Mining) competition. The training dataset of the dataset contains seven weeks of network flow and about five million connection data, and the test dataset contains about two million data, including two weeks of network flow. There are normal and abnormal network data in the KDD99 dataset. The abnormal data contains four major types of attacks, which can be divided into 39 modes of attack, 22 of which are included in the training set and 17 are included in the test set. The four major types of attacks are: (1) DOS (Denial of Service attack), (2) U2R (User to Root), (3) R2L (Remote to Local), and (4) Surveillance or probe.

\subsection{Data Processing}

The KDD99 dataset contains more than five million network connection information, and its size is 708MB. For the current experiment, the amount of information is too large; therefore, it is necessary to select and deal with it. Usually, a $10 \%$ subdataset of the dataset is selected as experimental data. The dataset of KDD 99 contains 41-dimensional feature attributes. In order to facilitate the experiment, the attributes that have little effect on intrusion detection are removed and ten attributes 
are selected for the experiment. These ten attributes are: duration, protocol-type, service, flag, src_bytes, dst_bytes, count, srv count, dst_host_count, and dst_host_srv_count.

We used the 10800 records in $10 \%$ subdatasets as the experimental data, including 10000 normal data records and 800 attack data records. The data were normalized according to the normalization formula. The selected data were divided into two classes, normal class and abnormal class, and the abnormal class contains four major modes of attack. For the experiment, the data records were divided into three datasets. Dataset-1 contains 1000 training samples and 500 test samples, dataset- 2 contains 2500 training samples, and dataset-3 contains 3700 training samples and 2000 test samples.

\subsection{Analysis of Results}

Firstly, it is necessary to verify the accuracy of classification of the improved algorithm after optimizing the parameters of SVM, and the training data is selected as 60,95 , and 130 respectively.

Figure 5 below shows the experimental results of the improved algorithm. By improving the quantum and immune algorithm, in this experiment, when the sample number is 60 , the difference between the highest and lowest classification accuracy is $0.78 \%$; when the sample number is 95 , the difference is $0.76 \%$; and when the sample number is 130 , the difference is $0.64 \%$. The classification effect of the improved algorithm combined with SVM is better. Both the highest and the lowest classification accuracy are improved, and the classification results are consistent. The improved algorithm overcomes the premature convergence to some extent and improves the performance of the whole algorithm.

The following is the application experiment of the improved algorithm in the network intrusion detection. Taking the experimental results of dataset-2 as an example, the parameters optimization algorithms of SVM based on grid searching, the immune algorithm, the quantum and immune algorithm, and the improved algorithm are compared, and the experimental results are shown in Figures 6 and 7.

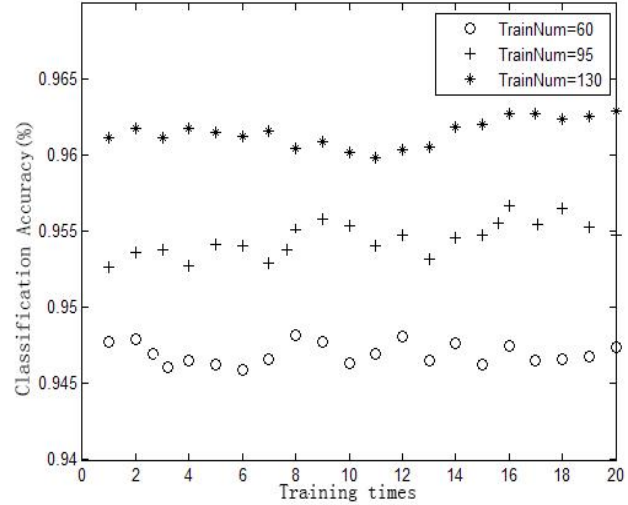

Figure 5. The accuracy of the improved algorithm for different sample sizes

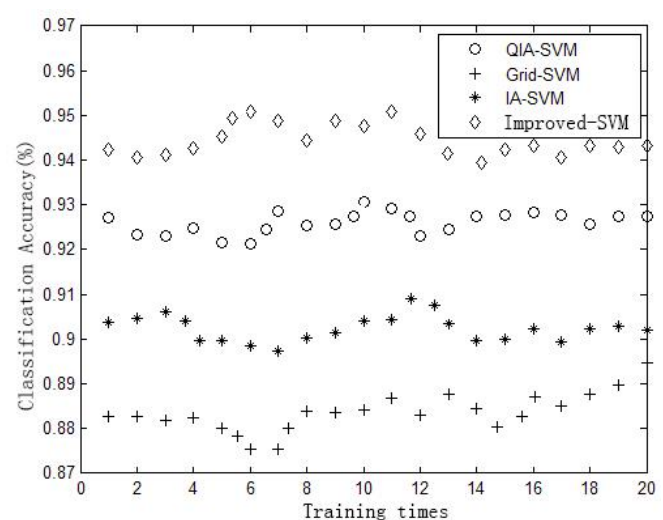

Figure 6. Classification accuracy of the four kinds of algorithm

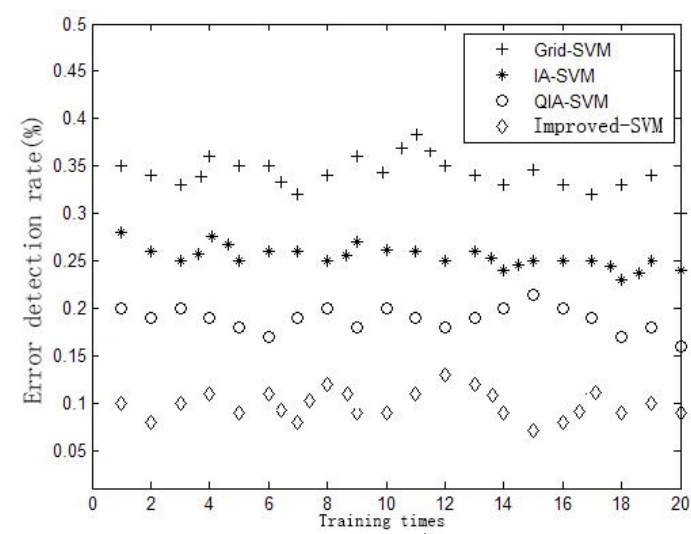

Figure 7. Error detection rate of the four kinds of algorithm

In dataset-2, each algorithm was trained 20 times, and the results of each algorithm are represented by the two figures. We can see clearly from Figures 6 and 7 that the classification accuracy of SVM based on the improved algorithm is higher 
than that of other algorithms, and its false detection rate is lower than that of other algorithms. The improved quantum and immune algorithm can avoid individual retrogression in evolution, improve the performance of the algorithm, and then improve the classification accuracy of SVM.

Table 1 shows the performance metrics evaluated by precision, recall, and F-measure of the four algorithms on three datasets. Precision is the ratio of the number of relevant records classified to the total number of irrelevant and relevant records. Recall is the ratio of the number of relevant records classified to the total number of records in the dataset. Fmeasure is a statistical technique for determining accuracy based on both precision and recall.

\begin{tabular}{|c|c|c|c|c|c|c|c|c|c|}
\hline \multirow{2}{*}{ Algorithms } & \multicolumn{3}{|c|}{ Dataset1 } & \multicolumn{3}{|c|}{ Dataset2 } & \multicolumn{3}{|c|}{ Dataset3 } \\
\hline & Precision & Recall & F-measure & Precision & Recall & F-measure & Precision & Recall & F-measure \\
\hline $\begin{array}{l}\text { Grid search } \\
\text { algorithm }\end{array}$ & 90.84 & 92.23 & 91.55 & 89.62 & 93.10 & 92.21 & 92.53 & 93.76 & 91.35 \\
\hline $\begin{array}{l}\text { Immune } \\
\text { algorithm }\end{array}$ & 90.53 & 93.87 & 92.06 & 93.16 & 94.16 & 93.16 & 92.62 & 95.01 & 93.01 \\
\hline $\begin{array}{l}\text { Quantum and } \\
\text { immune } \\
\text { algorithm }\end{array}$ & 94.36 & 95.43 & 96.62 & 95.77 & 97.59 & 97.21 & 96.85 & 97.13 & 97.13 \\
\hline $\begin{array}{l}\text { The improved } \\
\text { algorithm }\end{array}$ & 97.44 & 98.27 & 98.27 & 99.02 & 99.02 & 99.02 & 98.67 & 99.01 & 98.99 \\
\hline
\end{tabular}

The experimental results on the three datasets show that the classification accuracy, recall and F-measure of the improved quantum and immune algorithm combined with SVM is better than those of other algorithms, and the false detection rate is also relatively lower. To sum up, the improved quantum and immune algorithm has some advantages, through the design of the fitness function and the improvement of the quantum rotate gate, to avoid premature convergence and individual retrogression in the evolution of the algorithm. Thus, the classification accuracy of the algorithm is improved, the false detection rate is reduced, and great results are obtained. The validity of the improved algorithm is proven.

\section{Conclusions}

In view of the research on parameters optimization methods of SVM, this paper proposed the parameter optimization algorithm of SVM based on the quantum and immune algorithm. Through the parallelism of the quantum and immune algorithm, the convergence speed of the algorithm is improved, and the algorithm is verified by experiments. Moreover, the problem of premature convergence and randomness and blindness in the collapse process of the proposed algorithm is improved in two ways. The experimental results show that the performance of SVM is improved, which shows that the proposed algorithm can optimize the parameters of SVM effectively.

\section{Acknowledgements}

This work was supported by the National Natural Science Foundation Project (No. 61472271).

\section{References}

1. C. Cortes and V. Vapnik, "Support Vector Networks," Machine Learning, Vol. 20, pp. 273-293, 1995

2. J. Y. Liu, H. X. Wang, Y. Y. Sun, and L. Li, "Adaptive Niche Quantum-Inspired Immune Clonal Algorithm," Natural Computing, Vol. 15, No. 2, pp. 297-305, 2016

3. L. Demidova, E. Nikulchev, and Y. Sokolova, "The SVM Classifier based on the Modified Particle Swarm Optimization," International Journal of Advanced Computer Science and Applications, Vol. 7, No. 2, pp. 16-24, 2016

4. S. T. Ikram, "Improving Accuracy of Intrusion Detection Model using PCA and Optimized SVM," Journal of Computing and Information Technology, Vol. 24, No. 2, pp. 133-148, 2016

5. G. P. Wang, S. Y. Chen, and J. Liu, "Anomaly-based Intrusion Detection using Multiclass-SVM with Parameters Optimized by PSO," International Journal of Security \& its Applications, Vol. 9, No. 6, pp. 227-242, 2015

6. X. Rong, J. Yan, and H. X. Guo, "An Effective Classification Method for BCI based on Optimized SVM by GA," in Proceedings of the $3^{\text {rd }}$ Global Congress on Intelligent Systems, pp. 3-6, 2012

7. Y. Liu and J. Du, "Parameter Optimization of the SVM for Big Data," in Proceedings of International Symposium on Computational Intelligence and Design, pp. 341-344, 2016

8. Y. Yuan, L. I. Kang, and A. H. Zhou, "Study on the Classification Method of Igneous Rocks based on PCA-GA-SVM," Mathematics in Practice and Theory, 2017

9. H. Mei, L. Yin, D. Liu, et al., "Analogue Circuit Fault Diagnosis based on SVM Optimized by IPSO," Journal of Electronic Measurement and Instrumentation, 2017

10. G. Steve, "Support Vector Machines for Classification and Regression,” ISIS Technical Report, 1998 
11. L. Zhao, Z. Y. Li, and D. Chen, "Quantum-Inspired Immune Algorithm for Intrusion Detection Problem," Computer Engineering and Applications, Vol. 47, No. 11, pp. 98-101, 2011

12. W. Ren, "Optimization Algorithm for Vehicle Scheduling Problem based on Quantum Immune," Computer Science, Vol. 40, No. 5, pp. 233-216, 2013

Yuling Tian is a doctor and Master's tutor in the College of Information and Computer at Taiyuan University of Technology. Her research interests include artificial intelligence and fault diagnosis. 